\title{
The Multi-Objective Optimal Design of the Disc Type Piezoelectric Motor with Stability Constraint*
}

\author{
Ting-Nung SHIAU**, Chung-Hao KANG**, De-Shin LIU** \\ and Tyau-Her YOUNG*** \\ ${ }^{* *}$ National Chung Cheng University, \\ 621 Chia Yi, Taiwan, R.O.C \\ E-mai: imetls@ccu.edu.tw \\ ***National Taiwan University of Science and Technology, \\ 106 Taipei, Taiwan, R.O.C.
}

\begin{abstract}
A verified hybrid optimization procedure, which combines the genetic algorithm (GA) with traditional optimization methods, is presented in this paper with a disc type piezoelectric motor. The optimization objectives include minimizing the frictional loss of the rotor and maximizing the efficiency and spin speed of the piezoelectric motor. The behavior constraints include spin speed, natural frequency, and stability. The Runge-Kutta method is applied to determine the dynamic response of this motor, and the Floquet theory is employed to analyze the motor's stability. The optimization algorithm (hybrid genetic algorithm, HGA) applies GAs to provide an initial design variables set, thereby avoiding the trial process; thereafter, traditional algorithms are employed to determine optimum results. With the single-objective and multi-objective optimizations by using the HGA, both the efficiency and spin speed of the motor can be improved, and the frictional loss of the coating can be decreased effectively, either individually or simultaneously. The designs of the designated performances can be derived as well.
\end{abstract}

Key words: Piezoelectric Motor, Optimization, Nonlinear Dynamic, Genetic Algorithms, Traditional Optimization Method, Stability Analysis

\section{Introduction}

The piezoelectric motor has been developed for many years but was not applied extensively until in recent years. The piezoelectric motor is one type of actuator that differs from traditional actuators. The thrust from the piezoelectric motor is the result of the vibration that occurs during the transformation of electrical energy to mechanical energy. The thrust employs friction to propel the rotor by the converse piezoelectric effect of the piezoelectric ceramic.

Several savants independently had the idea of this kind of piezoelectric motor. In this paper, the piezoelectric motor is considered as a disc type one; the disc type of the piezoelectric motor generally consists of one rotor and one stator. Kurosawa and Ueha ${ }^{(1)}$ analyzed the contact relationship between the rotor and the stator and calculated the motor's dynamic behaviors by assuming the motor's vertical vibration as the motion of linear spring. Yamabuchi and Kagawa ${ }^{(2)}$ used the Finite Element Method to analyze the elastic structure of electric-materials. A rotary electric motor with no-symmetrical vibration modes was developed by Takano et al..$^{(3)}$, but the efficiency of this kind of motor was low. The piezoelectric motor composed of a twisting vibrator was designed by Kurosawa and Ueha ${ }^{(4)}$, which is highly efficient and can exercise in two directions. According to the 
study of Kentrao et al. ${ }^{(5)}$, the maximum torque is relative to the voltage input, and the optimum design must consider the normal contact force between the stator and the rotor. Assuming the existence of two vibration modes and a standing wave, Takano et al. ${ }^{(6)}$ analyzed the rotary ultrasonic motor. Nesbitt et al. ${ }^{(7)}$ substituted the mechanical modes and electric modes into the systematic equations of motion. They used the Rayleigh-Ritz assumed mode method to get a similar solution of the dynamic characteristics. Nicola et al. ${ }^{(8)}$ used piezoelectric film made of alloy nickel to make an ultrasonic motor and simulated the rotor model using the theory of thin plate vibration. The modeling of the contact at the stator-rotor interface was developed by Maol and $\mathrm{Cusim}^{(9)}$. Wen et al. ${ }^{(10),}{ }^{(11)}$ used experimentation to analyze the system control and the dynamic characteristic of a disc piezoelectric motor, while Bai et al. ${ }^{(12)}$ presented an ultrasonic motor where rotation speed is locked by the phase-velocity.

In the optimization of the piezoelectric motor, Flynn ${ }^{(13)}$ optimized the torque and power of the rotor separately by experiment. In addition to modeling the contact interface, Maol and $\mathrm{Cusim}^{(9)}$ also optimized the rotor architecture in their study. Ville and Amit ${ }^{(14)}$ simulated the performance with different piezo thicknesses and measured the torque and rotation rate with different electric inputs. Ishii et al. ${ }^{(15)}$ improved the efficiency of the motor by using lubricant. Pons et al. ${ }^{(16)}$ discussed the relationship between performance, stator geometry and mater parameter. Zhao et al. ${ }^{(17)}$ used the constrained variable metric algorithm for single and multi-objective optimization. For the stability analysis, Xia and Wang ${ }^{(18)}$ analyzed the rotor by experimenting with different factors, such as the driving frequency, the rotor's weight and radius, the mode number of stator vibration, and the fluid's height.

In this paper, the hybrid genetic algorithm (HGA) is applied to identify optimal designs of the piezoelectric motor with stability constraints. This hybrid optimization procedure first appeared in research by Shiau et al. ${ }^{(19)}$ With the hybrid procedure, the GA is applied to identify the initial design variables, first by searching widely over a large design space of the whole domain. As a second step, that adapted optimization algorithm is focused upon promising sub-regions to identify the best design in that region. This two-stage optimization procedure can reduce the trial process and effectively generate better design variables.

\section{Modeling approach of the piezoelectric motor}

The piezoelectric motor is considered as a disc type one in this paper; that the disc type of the piezoelectric motor generally consists of one rotor and one stator. In the following is the simple description of the disk type piezoelectric motor:

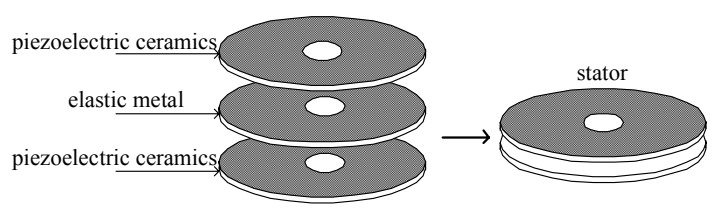

Fig. 1 The basic conformation of a stator ${ }^{(7)}$

\section{A. System formulation}

The principal part of the disc type piezoelectric motor consists of one stator and one rotor. As shown in Fig. 1, the stator is composed of two thin piezoelectric ceramic discs and one disc of elastic metal substrate between the two piezoelectric ceramic discs. Fig. 2 shows an interface model between the rotor and stator. In this model, an applied load forces the rotor to keep contact with the stator. The modeling approach and system equation formulation of the piezoelectric motor are given in Nesbitt's paper ${ }^{(7)}$. The equations of motion for the system are described as follows: 


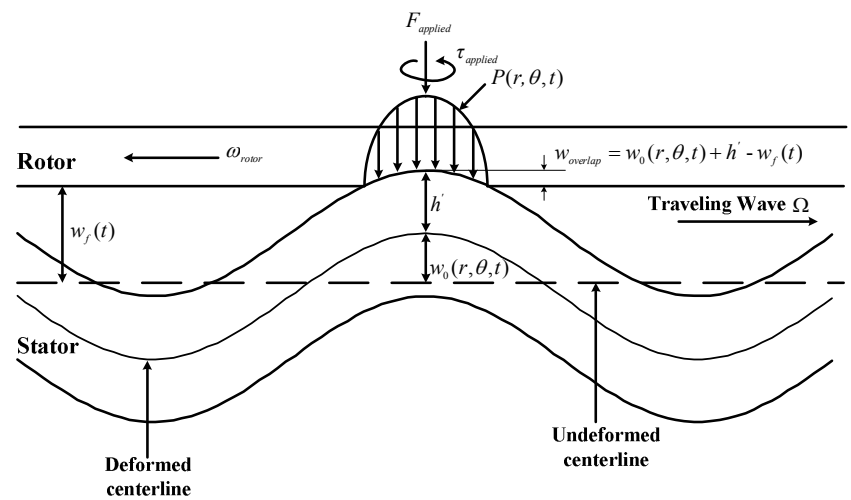

Fig. 2 A contact model between the rotor and the stator ${ }^{(7)}$

$$
\begin{aligned}
& {[M]\{\ddot{p}\}+[C]\{\dot{p}\}+[K]\{p\}=[\Theta]\{V\}+\left\{F_{N}\right\}+\left\{F_{T}\right\}} \\
& M_{r} \ddot{w}_{f}+C_{z} \dot{w}_{f}=F_{\text {int }}-F_{\text {applied }} \\
& I_{r} \ddot{\alpha}+C_{\alpha} \dot{\alpha}=\tau_{\text {int }}-\tau_{\text {applied }}
\end{aligned}
$$

where $[M],[C],[K]$ and $[\Theta]$ are system mass, damping, stiffness and electro mechanical matrixes; $\{p\}$ is the mechanical modal amplitude; $\{V\}$ is the input voltage; $\left\{F_{N}\right\}$ and $\left\{F_{T}\right\}$ are modal normal and tangential forces; $M_{r}, C_{Z}, I_{r}$ and $C_{\alpha}$ are the mass, vertical damping, inertia and spin damping of rotor; $w_{f}, F_{\text {int }}$ and $\tau_{\text {int }}$ are the separation, interface force and torque between the rotor and stator; $\alpha$ is the spin angle of the rotor; $F_{\text {applied }}$ is the applied axial and $\tau_{\text {applied }}$ is the applied torque. The average output power can be calculated as

$$
P_{\text {out }}=\tau_{\text {applied }} \omega_{\text {rotor }}
$$

and the total input power is defined as

$$
P_{i n}=V\left(\Theta^{T} \dot{p}+C_{p} \dot{V}\right)
$$

The motor efficiency is expressed as the ratio of the output to the input power.

$$
\eta=P_{\text {out }} / P_{\text {in }} \times 100 \%
$$

\section{B. Stability analysis}

In order to achieve the stability analysis by the Floquet theory, Eq. (1) (3) need to be expressed as where

$$
[\bar{M}]\{\ddot{q}\}+[\bar{C}]\{\dot{q}\}+[\bar{K}]\{q\}=\{\bar{Q}\}+\{\bar{F}(t, q, \dot{q})\}
$$

$$
\begin{aligned}
& {[\bar{M}]=\left[\begin{array}{ccc}
{[M]} & 0 & 0 \\
0 & M_{r} & 0 \\
0 & 0 & I_{r}
\end{array}\right],[\bar{C}]=\left[\begin{array}{ccc}
{[C]} & 0 & 0 \\
0 & C_{z} & 0 \\
0 & 0 & C_{\alpha}
\end{array}\right],[\bar{K}]=\left[\begin{array}{ccc}
{[K]} & 0 & 0 \\
0 & 0 & 0 \\
0 & 0 & 0
\end{array}\right],} \\
& \{q\}^{T}=\left\{\{p\}^{T}, w_{f}, \alpha\right\},\{\bar{Q}\}^{T}=\left\{[\Theta]\{V\},-F_{\text {applied }},-\tau_{\text {applied }}\right\} \text { and }\{\bar{F}\}^{T}=\left\{F_{N}+F_{T}, F_{\text {int }}, \tau_{\text {int }}\right\}
\end{aligned}
$$

In the above, $\{\bar{Q}\}$ and $\{\bar{F}\}$ are time dependent ${ }^{(7)}$. According to the Floquet theory:

$$
\{y(t+T)\}=[Q(T)]\{y(t)\} \quad \text { where }\{y\}=\{q \quad \dot{q}\}^{T}
$$

$[Q(T)]$ is the Floquet transition matrix and is defined as

$$
[Q(T)]=\prod_{i=1}^{n-1}\left[\begin{array}{cc}
H & J \\
\frac{2}{\Delta t}(H-I) & \frac{2}{\Delta t} J-I
\end{array}\right]
$$

where

$$
[J]=D^{-1}\left(\frac{4}{\Delta t}[\bar{M}]_{i+1}+[\bar{D}]_{i+1}-[\bar{M}]_{i+1}[\bar{M}]_{i}^{-1}[\bar{D}]_{i}\right)
$$




$$
\begin{aligned}
& {[H]=D^{-1}\left(\frac{4}{(\Delta t)^{2}}[\bar{M}]_{i+1}+\frac{2}{\Delta t}[\bar{D}]_{i+1}-[\bar{M}]_{i+1}[\bar{M}]_{i}^{-1}[\bar{K}]_{i}\right)} \\
& {[\bar{D}]_{i+1}=[\bar{C}]_{i+1}-\left[Q_{2}\right]_{i+1} \text {, and }[\bar{K}]_{i+1}=[\bar{K}]_{i+1}-\left[Q_{2}\right]_{i+1}}
\end{aligned}
$$

then

$$
\operatorname{det}([Q(T)]-\Lambda[I])=0 \quad \text { where } \Lambda_{i}=e^{\eta_{1} T}=A_{i}+j B_{i}
$$

The stability criterion for the system can be expressed by $\left\|\Lambda_{i}\right\|^{2}=A_{i}^{2}+B_{i}^{2}<1$ or $\sigma_{i}<0$; where $\|\Lambda\|$ is called the stability index. If the system stability index $\|\Lambda\|$ is smaller than 1 , the system is stable. On the contrary, if the system is considered unstable the $\|\Lambda\|$ will be lager than 1 .

\section{Program of optimization design}

The objective of the optimization approach given in this paper includes maximizing the efficiency and spin speed of the rotor and minimizing the frictional loss of the coating that is under the rotor. Maximization and minimization can be separately or simultaneously. In order to reduce frictional loss, minimizing the maximum value of $w_{\text {overlap }}$ in steady state becomes one of the objectives. The objective functions are shown in Eq. (14). The design variables, such as the inner radius $r_{\text {inner }}$; outer radius $r_{\text {outer }}$; thickness of the substrate $t_{s}$; thickness of the piezoelectric ceramic $t_{p}$, and the friction coefficient of the coating $\mu$, are chosen to be the geometric parameters of the piezoelectric motor. In order to produce the resonance of the piezoelectric effect by using the similar voltage frequency and keep the motor system is stable; the behavior constraints are instituted as Eq. (15):

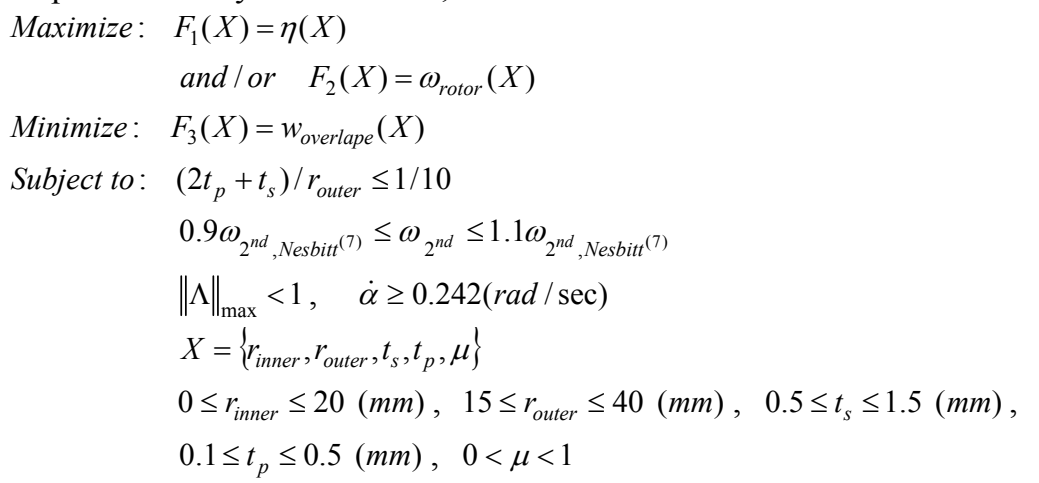

where $r_{\text {inner }}$ is the inner radius; $r_{\text {outer }}$ is the outer radius; $t_{s}$ is the substrate thickness; $t_{p}$ is the piezo thickness; $\mu$ is the friction coefficient; $\eta$ is the motor efficiency; $\dot{\alpha}$ is the rotor spin speed; $w_{\text {overlap }}$ is the contact value between the rotor and stator; $\omega_{2^{n d}}$ is the second natural frequency; and $\|\Lambda\|$ is the stability index.

\section{Description of the hybrid genetic algorithm}

Most traditional optimization methods require a better set of initial values for the design variables, and then converge rapidly to generate good results. However, most of them face the same difficulty, such as a long trial-and-error process in finding a better set of initial design variables. How to select better initial values of the design variables is a critical step for those methods. As for using the GA, it has the advantage of starting in a random population. Even though a GA can locate the solution in the whole domain, it does not solve constraint problems easily, especially for exact constraints. In this paper, the HGA is applied to overcome these difficulties and identify optimal designs of the piezoelectric motor with stability constraints. This kind of two-stage optimization procedure can reduce the trial process and effectively generate better design variables. In the first step, the GA is applied to provide a set of initial design variables, which are then used in conjunction with traditional algorithms to determine the optimum results. Fig. 3 is the flow chart depicting the HGA, and the procedures are described as follows: 


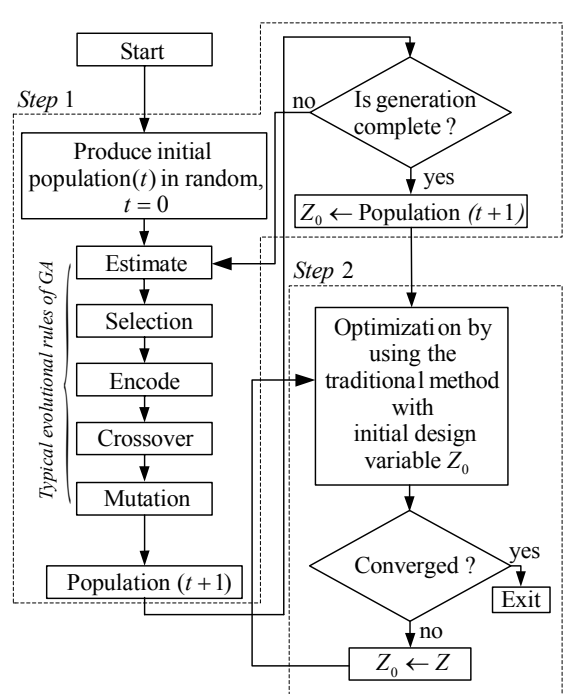

Fig. 3 Flow chart of the Hybrid Genetic Algorithm

Step 1: Calculate the set of initial design variables by genetic algorithm.

In the HGA, the typical GA evolutional rules are applied to calculate the set of initial variables in the first step. GA represents a novel stochastic method for solving optimization problems without initial design variables. The cost function generates an output form a set on input variables. In this paper, Eq. (14) is considered as the fitting function in the first step of HGA optimization process. The GA begins by defining a chromosome or an array of variable values to be optimized. The inner radius; outer radius; substrate thickness; piezo thickness and friction coefficient are selected as the design variables. Those design variables are considered genes, which are made up of chromosomes, and each chromosome is a coded representation of a design variable. The code in this study is a binary string, and each bit represents a gene. An example of binary encoded chromosome that has $N_{\text {var }}$ variables, each encoded with $N_{\text {gene }}=10$ bits, is

$$
\text { chromosome }=[\underbrace{11110010010011011111}_{\text {gene }_{1}} \cdots \underbrace{0000101001}_{\text {gene }_{2}}]
$$

where gene $=\left[\begin{array}{llll}b_{1} & b_{2} & \cdots & b_{N_{\text {gene }}}\end{array}\right] ; N_{\text {gene }}$ is the number bits in a gene; $b_{n}$ is the binary bit ( 1 or 0 ). The GA starts with an initial random population and the upper and lower boundaries of the initial random design variables are shown in Eq. (15). Successive populations are produced, primarily by the operations of estimation, selection, encoding, crossover, and mutation. The detailed description of the GA can be found in the following

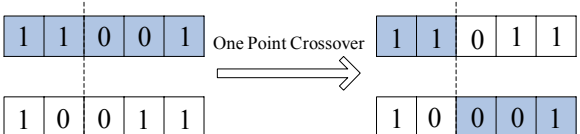

(a) Crossover

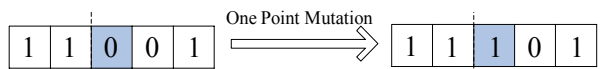

(b) Mutation

Fig. 4 One point crossover and mutation of the binary chromosome references ${ }^{(20 \sim 23)}$. Estimation is the operator that appraises the merit of the chromosome and the corresponding cost function in the evolutional process. The purpose of operator selection in a GA is to give more reproductive chances, one whole, to those population members that are the most fit. There are many ways to do this, and one commonly used technique is fitness-proportional, or roulette wheel parent selection. The roulette-wheel is selected as the selection method in this paper. Crossover recombines the traits of the selected members in the hope of producing an offspring with a better fitness level than its parents. Holland ${ }^{(21)}$ experimented with a crossover operator called one-point crossover, which is applied in this HGA. One-point crossover occurs when parts of two parent chromosomes are swapped after a randomly selected point, creating two children. Fig. 
4(a) shows the crossover of the binary chromosome, the children are made by cutting the parents at the point denoted by vertical line and exchanging parental genetic material after the cut. After crossover, the next process is mutation. Some GA practitioners use bit mutation to flip bits. Bit mutation is one procedure carried out by one-point mutate. When bit mutation is applied to a bit string it sweeps down the list of bits, replacing each by a randomly selected bit if a probability test is passed. Fig. 4(b) shows the mutation of the binary chromosome. After the successive operations, the pre-set numbers of generation and population are ascertained to see whether that they are completed or not. In this paper, the numbers of generation and population are selected as 500 and 1000 . The optimal design variables, which are determined using the GA, replaced the initial design variable with the trial-and-error process.

\section{Step 2: Determine the optimum results using a mathematical programming method.}

In step 2, once the initial design variables have been decided, the mathematical programming method is applied to determine the optimum results. Three optimization algorithms ${ }^{(24)}$, which include the goal programming method (GPM), the sequential quadratic programming (SQP) and the augmented Lagrange multiplier method (ALMM), are employed individually for the optimization design in this paper:

(i) For the goal programming method (GPM), the objectives are driven to attain the prescribed goals that the designer wishes to achieve. Thus, the solution $Z^{*}$ is defined as the one that minimizes deviations from the goals. Mathematically, it is expressed as follows:

$$
\begin{aligned}
& \text { Minimization : }\left[\sum_{i=1}^{N_{0}}\left(\alpha_{i}^{+}+\alpha_{i}^{-}\right)^{P}\right]^{1 / P}, P \geq 1 \\
& \text { Subject to : } Z \in C \\
& F_{i}-\alpha_{i}^{+}+\alpha_{i}^{-}=b_{i}, \quad i=1 \cdots N_{0} \\
& \alpha_{i}^{+}, \alpha_{i}^{-} \geq 0, \quad \alpha_{i}^{+} \cdot \alpha_{i}^{-}=0
\end{aligned}
$$

where the corresponding set of design vector $Z$ is defined by $C ; N_{0}$ is the number of objectives; $b_{i}$ is the goal for the $i^{\text {th }}$ objective; and $\alpha_{i}^{+}, \alpha_{i}^{-}$are the under and over achievement of the $i^{\text {th }}$ goal, respectively. The value of $P$ is chosen to be '2'; $F_{i}$ is the value of the $i^{t h}$ objective function; and the goal $b_{i}$ is set to be $F_{i}^{*}$, which is the optimum value for the case of a single objective design.

(ii) The quadratic programming sub-problem is solved unceasingly using sequential quadratic programming (SQP), which begins by determining the desired search direction. The SQP creates a quadratic approximation of the augmented objective function and a linear approximation of the constraints. The direction-finding problem is expressed as

$$
\begin{aligned}
& \text { Minimization: } Q(S)=F(Z)+\nabla F(Z) \cdot S+\frac{1}{2} S^{T} B S \\
& \text { Subject to: } \nabla g_{k}(Z) \cdot S+\delta_{k} g_{k} \leq 0, \quad k=1 \cdots m
\end{aligned}
$$

where $S$ is the search direction; $g_{k}(Z)$ is the inequality constraint; and $m$ is the number of inequality constraints. The matrix $B$ is a positive definite matrix, which is initially the identity matrix, and is updated on subsequent iterations. The scalar parameter $\delta_{k}$ is defined as

$$
\delta_{k}=\left\{\begin{array}{lll}
1 & \text { if } & g_{k}(Z)<0 \\
\bar{\delta} & \text { if } & g_{k}(Z) \geq 0
\end{array}\right.
$$

The parameter $\bar{\delta}$ should be chosen as near to '1' as possible. Typically, $\bar{\delta}=0.9$ to 0.95 works well.

(iii) The augmented Lagrange multiplier method (ALMM), which combines the Lagrange multiplier method with the penalty function method, is used to dispose of behavior constraints.

Minimization: $A\left(Z, \lambda, r_{p}\right)$ 
Subject to : $g_{k}(Z) \leq 0, \quad k=1 \cdots m$

The Lagrangian is created as

$$
L(Z, \lambda)=F(Z)+\sum_{k=1}^{m} \lambda_{k} g_{k}(Z)
$$

and $A\left(Z, \lambda, r_{p}\right)=F(Z)+\sum_{k=1}^{m}\left\{\lambda_{k}\left[g_{k}(Z)+S_{k}^{2}\right]+r_{p}\left[g_{k}(Z)+S_{k}^{2}\right]^{2}\right\}$

The pseudo-objective function given in Eq. (9) is referred to as the augmented Lagrangian, which combines the Lagrange function with the penalty function, where $\lambda_{k}$ and $r_{p}$ are the multipliers that determine the magnitude of the penalty, and $S_{k}$ is the added slack variable, that gives:

$$
g_{k}(Z)+S_{k}^{2}=0
$$

This kind of two-stage optimization procedure can reduce the trial process and effectively generate better design variables. In the following section, this hybrid procedure is applied to the optimal design of the disc type piezoelectric motor with stability constraint.

\section{Numerical results and discussion}

Three examples, including single-objective and multi-objective optimizations, are used to demonstrate the merits of the design algorithm. The effects of system performances with different non-dimensional design variables in the design range are shown in Fig. 5. In Fig. 5, the horizontal axes are the non-dimensional $r_{\text {inner }}, r_{\text {outer }}, t_{s}, t_{p}$ and $\mu$ with different line forms individually. Where the non-dimensional $r_{\text {inner }}=\left(r_{\text {inner }}-0\right) /(20-0)$, non-dimensional $r_{\text {outer }}=\left(r_{\text {outer }}-15\right) /(40-15)$, non-dimensional $t_{s}=\left(t_{s}-0.5\right) /(1.5-0.5)$, non-dimensional $t_{p}=\left(t_{p}-0.1\right) /(0.5-0.1)$ and non-dimensional $\mu=\mu$. Changing these design variables results in conspicuous variations of system performances. The system parameters and performances of the piezoelectric motor in Nesbitt's paper ${ }^{(7)}$ are shown in Table 1. With the stability analyzed by the Floquet theory, the motor system in Nesbitt's study is possibility unstable.

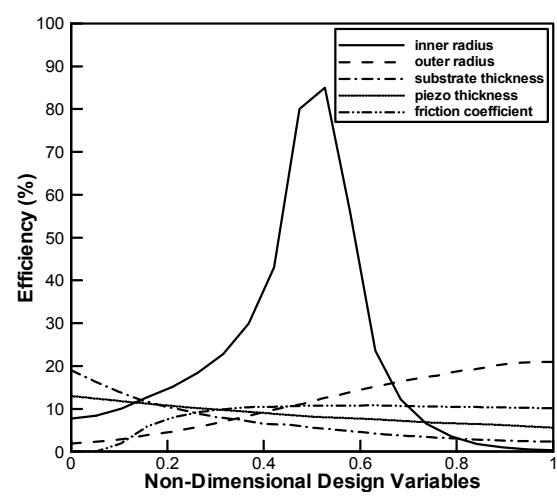

(a)

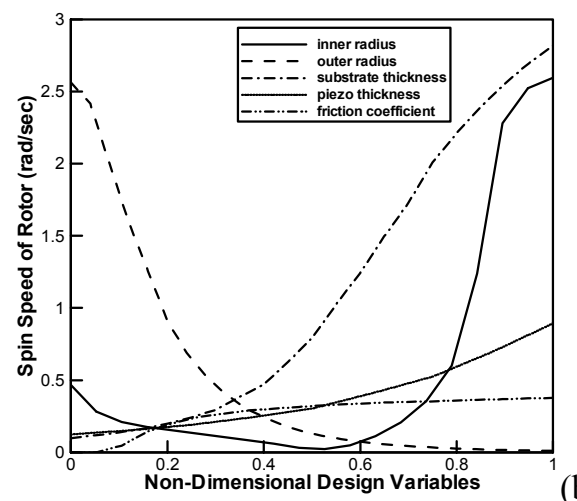

(b)

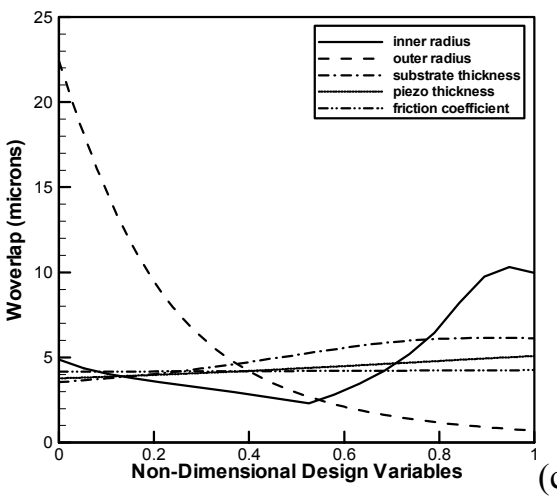

Fig. 5 The effects on performance with different non-dimensional design variables 
(i) Example 1: Single-objective optimization of the piezoelectric motor

In this example, the objective includes motor efficiency, rotor spin speed, and frictional loss of the coating, individually. As Table 2 shows, the spin speed of the rotor can be increased substantially. But with the optimization, motor efficiency is reduced, and the maximum value of $w_{\text {overlap }}$ in steady state is increased at the same time. The reason for this is that, with the optimized design the input power increases. Even though the increase in the friction force increases the spin speed, the friction force between the rotor and stator also increases both the power loss of the motor and the frictional loss of the coating. With

Table 1 The design variables and system performances in Nesbitt's paper ${ }^{(7)}$

\begin{tabular}{llll}
\hline \multicolumn{3}{c}{ Nesbitt,1995 } \\
\hline \multicolumn{1}{c}{ Design } & \multicolumn{2}{c}{ Variables } & \multicolumn{2}{c}{ System Performances } \\
\hline Inner Radius $(\mathrm{cm})$ & 0.25 & Efficiency $(\%)$ & 7.5298403 \\
Outer Radius $(\mathrm{cm})$ & 2.50 & Spin Speed $(\mathrm{rad} / \mathrm{sec})$ & 0.3069750 \\
Substrate Thickness $(\mathrm{mm})$ & 0.75 & $w_{\text {overlap }}$ (microns) & 4.20 \\
Piezo Thickness $(\mathrm{mm})$ & 0.25 & $4^{\text {th }}$ Natural Frequency $(\mathrm{Hz})$ & 8343.7282 \\
Friction Coefficient & 0.30 & $\|\Lambda\|$ & 1.00000028 \\
\hline
\end{tabular}

Table 2 Single-objective optimization of rotor spin speed

\begin{tabular}{|c|c|c|c|c|}
\hline & \multicolumn{4}{|c|}{ Hybrid Genetic Algorithm } \\
\hline & Initial & GPM & SQP & ALMM \\
\hline & Design & Variables & & \\
\hline Inner Radius $(\mathrm{cm})$ & 0.00706 & 0.00706 & 0.00707 & 0.00706 \\
\hline Outer Radius $(\mathrm{cm})$ & 2.99719 & 3.04570 & 3.01486 & 3.01940 \\
\hline Substrate Thickness $(\mathrm{mm})$ & 1.14146 & 1.17265 & 1.16265 & 1.15101 \\
\hline Piezo Thickness $(\mathrm{mm})$ & 0.27989 & 0.28263 & 0.28029 & 0.29263 \\
\hline Friction Coefficient & 0.98522 & 0.97563 & 0.95634 & 0.98522 \\
\hline \multicolumn{5}{|c|}{ System Performances } \\
\hline Efficiency $(\%)$ & 1.28557 & 1.19143 & 1.22599 & 1.22898 \\
\hline Spin Speed $(\mathrm{rad} / \mathrm{sec})$ & 2.91973 & 2.95558 & 2.96264 & 2.96796 \\
\hline$w_{\text {overlap }}$ (microns) & 5.28592 & 5.13528 & 5.22185 & 5.22288 \\
\hline $4^{\text {th }}$ Natural Frequency $(\mathrm{Hz})$ & 9177.78 & 9104.71 & 9212.65 & 9176.57 \\
\hline$\|\Lambda\|$ & 0.99955 & 0.99996 & 0.99974 & 0.99995 \\
\hline
\end{tabular}

this HGA, the GA can determine an optimum set of design variables; the traditional algorithms, GPM, SQP, and ALMM, just increase the spin speed of the motor with smaller values after the GA optimization. Table 3 shows that the motor efficiency is effectively increased. For the same reason as given above, the rotor spin speed is reduced, and $w_{\text {overlap }}$ is increased in the process of optimizing motor efficiency. As Table 3 shows, the HGA combining GA with ALMM has the most ability when in contrast with the GPM and SQP in the increasing of the motor efficiency. In the optimization of frictional loss, minimizing the maximum value of $w_{\text {overlap }}$ in steady state is the objective. The optimum design is shown in Table 4. In Table 3 and Table 4, even the rotor spin speed sometimes becomes very close to the lowest bound of the behavior constraints. With the stability 
analysis, all the stability indices $\|\Lambda\|$ of the single-objective optimization are less than 1 . This shows that the piezoelectric motor is stable after the optimum design but is on the verge of being unstable.

(ii) Example 2: Multi-objective optimization of the piezoelectric motor

Two optimization objectives, including the maximization of motor efficiency and rotor spin speed, are presented for the Pareto multi-objective optimization. The weighting objectives method (Charnes and Cooper ${ }^{(25)}$ ) is applied to solve this Pareto optimum Table 3 Single-objective optimization of motor efficiency

\begin{tabular}{lcccc}
\hline & \multicolumn{2}{c}{ Hybrid Genetic Algorithm } & \\
\cline { 2 - 5 } & Initial & GPM & SQP & ALMM \\
\hline & Design & Variables & & \\
\hline Inner Radius $(\mathrm{cm})$ & 0.55435 & 0.58046 & 0.60047 & 0.61473 \\
Outer Radius $(\mathrm{cm})$ & 1.95847 & 1.84257 & 1.90555 & 1.88680 \\
Substrate Thickness $(\mathrm{mm})$ & 0.51764 & 0.50467 & 0.52467 & 0.50509 \\
Piezo Thickness $(\mathrm{mm})$ & 0.10363 & 0.12550 & 0.10550 & 0.11368 \\
Friction Coefficient & 0.58315 & 0.59157 & 0.57157 & 0.51011 \\
\hline & System Performances & & \\
\hline Efficiency $(\%)$ & $\mathbf{2 9 . 1 3 3 2}$ & $\mathbf{3 0 . 1 5 8 9}$ & $\mathbf{3 1 . 9 7 7 0}$ & 36.3478 \\
Spin Speed $(\mathrm{rad} / \mathrm{sec})$ & 0.24463 & 0.34555 & 0.26141 & 0.24310 \\
$w_{\text {overlap }}($ microns $)$ & 7.23168 & 8.95750 & 7.82662 & 8.02437 \\
$4^{\text {th }}$ Natural Frequency $(\mathrm{Hz})$ & 8245.98 & 9372.75 & 8924.46 & 8893.59 \\
& 0.99999 & 0.99992 & 0.99988 & 0.99999 \\
\hline$\|\Lambda\|$ & & & &
\end{tabular}

Table 4 Single-objective optimization of $w_{\text {overlap }}$

\begin{tabular}{lcccc}
\hline & \multicolumn{3}{c}{ Hybrid Genetic Algorithm } & \\
\cline { 2 - 5 } & Initial & GPM & SQP & ALMM \\
\hline & Design & Variables & & \\
\hline Inner Radius $(\mathrm{cm})$ & 0.62469 & 0.54165 & 0.60466 & 0.49906 \\
Outer Radius $(\mathrm{cm})$ & 2.97556 & 3.05645 & 3.03547 & 3.10570 \\
Substrate Thickness $(\mathrm{mm})$ & 1.34079 & 1.25683 & 1.13469 & 1.15630 \\
Piezo Thickness $(\mathrm{mm})$ & 0.22902 & 0.24596 & 0.26568 & 0.29561 \\
Friction Coefficient & 0.43729 & 0.67490 & 0.76559 & 0.74568 \\
\hline & System Performances & & \\
\hline Efficiency $(\%)$ & 6.67188 & 7.12880 & 7.88601 & 7.55536 \\
Spin Speed $(\mathrm{rad} / \mathrm{sec})$ & 0.24273 & 0.25878 & 0.15237 & 0.24261 \\
$w_{\text {overlap }}$ (microns) & $\mathbf{2 . 2 9 2 9 1}$ & $\mathbf{2 . 2 4 1 0 6}$ & $\mathbf{2 . 0 0 8 4 2}$ & $\mathbf{2 . 1 6 4 4 9}$ \\
$4^{\text {th }}$ Natural Frequency $(\mathrm{Hz})$ & 9113.49 & 8252.52 & 7660.26 & 7647.84 \\
$\quad\|\Lambda\|$ & 0.99976 & 0.99987 & 0.99962 & 0.99932 \\
\hline
\end{tabular}


problem. Table 5 shows the multi-objective optimization results. Although after the optimization with GA, the stability index is larger than 1, the system is still stable after optimization with the hybrid method. In this optimization design process, motor efficiency is increased, but there is only less increase in the spin speed than the single-objective design in example 1. The reason is that it is not easy to improve both of the performances in the Pareto multi-objective optimization design process. With the same reason, neither the optimum motor spin speed nor efficiency by GA can be increased after Table 5 Multi-objective optimization of motor efficiency and rotor spin speed

\begin{tabular}{lcccc}
\hline & \multicolumn{2}{c}{ Hybrid Genetic Algorithm } & \\
\cline { 2 - 5 } & Initial & GPM & SQP & ALMM \\
\hline & Design & Variables & & \\
\hline Inner Radius $(\mathrm{cm})$ & 0.52151 & 0.52565 & 0.52457 & 0.52151 \\
Outer Radius $(\mathrm{cm})$ & 1.85422 & 1.84647 & 1.85367 & 1.85420 \\
Substrate Thickness $(\mathrm{mm})$ & 0.50779 & 0.50455 & 0.51046 & 0.50786 \\
Piezo Thickness $(\mathrm{mm})$ & 0.12927 & 0.12646 & 0.13265 & 0.12032 \\
Friction Coefficient & 0.71285 & 0.72466 & 0.70466 & 0.71236 \\
\hline & System & Performances & & \\
\hline Efficiency $(\%)$ & $\mathbf{2 3 . 8 9 9 9}$ & $\mathbf{2 4 . 5 4 0 8}$ & $\mathbf{2 3 . 4 9 2 5}$ & $\mathbf{2 4 . 4 1 5 8}$ \\
Spin Speed $(\mathrm{rad} / \mathrm{sec})$ & $\mathbf{0 . 4 3 0 0 1}$ & $\mathbf{0 . 4 2 4 7 3}$ & $\mathbf{0 . 4 3 8 0 6}$ & $\mathbf{0 . 4 1 0 2 4}$ \\
$w_{\text {overlap }}($ microns $)$ & 9.07970 & 9.17399 & 9.07534 & 9.03231 \\
$4^{\text {th Natural Frequency }(\mathrm{Hz})}$ & 9250.85 & 9256.98 & 9332.75 & 9172.71 \\
$\quad\|\Lambda\|$ & 1.00025 & 0.99976 & 0.99996 & 0.99994 \\
\hline
\end{tabular}

Table 6 Designated performances of motor efficiency, rotor spin speed, and $w_{\text {overlape }}$

\begin{tabular}{lccrr}
\hline & Example 3-1 & Example 3-2 & Example 3-3 \\
\hline & Designated Performances & \\
\hline Efficiency $(\%)$ & Non-designate & 10.0 & 5.0 \\
Spin Speed $(\mathrm{rad} / \mathrm{sec})$ & 2.0 & Non-designate & 1.0 \\
$w_{\text {overlap }}($ microns $)$ & 4.5 & 4.0 & 5.0 \\
\hline & Design & Variables & & \\
\hline Inner Radius $(\mathrm{cm})$ & 0.19065 & 0.64078 & 0.06938 \\
Outer Radius $(\mathrm{cm})$ & 2.97893 & 2.69080 & 2.66401 \\
Substrate Thickness $(\mathrm{mm})$ & 1.10085 & 0.91132 & 0.57652 \\
Piezo Thickness $(\mathrm{mm})$ & 0.41555 & 0.28661 & 0.49421 \\
Friction Coefficient & 0.94396 & 0.92807 & 0.85133 \\
\hline
\end{tabular}

System Performances

\begin{tabular}{llll}
\hline Efficiency (\%) & 1.98529 & $\mathbf{9 . 9 7 0 8 1}$ & $\mathbf{4 . 9 7 5 4 1}$ \\
\cline { 2 - 3 }
\end{tabular}




\begin{tabular}{llll}
\cline { 2 - 3 } Spin Speed $(\mathrm{rad} / \mathrm{sec})$ & $\mathbf{2 . 1 4 1 0 4}$ & 0.19763 & $\mathbf{0 . 9 4 4 6 0}$ \\
\cline { 2 - 4 }$w_{\text {overlap }}($ microns$)$ & $\mathbf{4 . 4 9 6 8 7}$ & $\mathbf{2 . 7 4 6 9 3}$ & $\mathbf{4 . 5 0 7 9 4}$ \\
\cline { 2 - 4 } $4^{\text {th }}$ Natural Frequency $(\mathrm{Hz})$ & 9175.10 & 8138.16 & 8829.31 \\
\cline { 2 - 4 }$\|\Lambda\|$ & 1.00052 & 0.99971 & 1.00072 \\
\hline
\end{tabular}

the HGA optimization design collectively. In Table 5, $w_{\text {overlap }}$ is raised; this means the frictional loss of the coating is increased substantially for the multi-objective optimization design.

For the multi-objective optimization design of the spin speed, motor efficiency, and $w_{\text {overlap }}$; it is not easy to determine the best design variables that would improve each objective at the same time, even if the numbers of the generation and population in GA is increased because the inverse proportion between those performances is conspicuous.

\section{(iii) Example 3: Designated Performances design of the piezoelectric motor}

In order to satisfy the requirement in engineering, some performances of the piezoelectric motor need to be designated. In example 3, the designs of the designated performances can be decided with the optimization design method and the designated performances can be chosen according to the engineering demands. In this example, the stability is not considered as the behavior constraint of the optimization. If the motor efficiency, spin speed, and $w_{\text {overlap }}$ are designated as $\bar{\eta}, \bar{\varpi}_{\text {rotor }}$ and $\bar{w}_{\text {overlap }}$, Eq. (23) can be expressed as follows:

$$
\begin{array}{rr}
\text { Minimize: } & F_{1}(X)=\left|\varpi_{\text {rotor }}-\bar{\varpi}_{\text {rotor }}\right| / \bar{\varpi}_{\text {rotor }} \\
& \text { and /or } \quad F_{2}(X)=|\eta-\bar{\eta}| / \bar{\eta} \\
\text { and } \quad w_{\text {overlap }}-\bar{w}_{\text {overlap }} \leq 0
\end{array}
$$

The results of the optimization design are shown in Table 6 and the designated performances can be achieved on condition that not all the performances are designated to be increased at the same time. The results show that the designated performances can be achieved within reasonable design ranges. If the optimization algorithm cannot determine the optimum design variables of the designated performances, it means that the values of the designated performances are infeasible. In other words, the designer can confirm the feasibility of the designated performances by using the optimization design method.

\section{Conclusion}

In this paper, a verified hybrid optimization design procedure (Hybrid Genetic Algorithm, HGA), which combines the genetic algorithm with traditional optimization methods, is presented with the optimization for a disc type piezoelectric motor under behavior constraints. According to the hybrid proposed method, the designer can get the superior design of the piezoelectric motor without determining the initial design as with traditional optimization methods. The results show that the efficiency and spin speed of a motor can be improved, individually and simultaneously, and the frictional loss of the coating can be diminished. With the designated performance of the rotor, the optimal design variables can also be found and fall within reasonable ranges for engineering requirements.

\section{References}

(1) Kurosawa, M. and Ueha, S., Efficiency of ultrasonic motor using traveling wave symmetrical structure, Journal of the Acoustical Society, Vol.44 (1988), pp.40.

(2) Yamabuchi, T. and Kagawa, Y., Numerical simulation of a piezoelectric ultrasonic motor and it's characteristics, Simulation, Vol.8-3 (1989), pp.69. 
(3) Takano, T., Horoshi, H. and Yoshiro, T., Analysis of nonaxisymmetric vibration mode piezoelectric annular plate and its application to an ultrasonic motor, IEEE Transactions on Ultrasonics, Ferroelectrics, and Frequency Control, Vol.37 (1990), pp.558-565.

(4) Kurosawa, M. and Ueha, S., Hybrid transducer type ultrasonic motor, IEEE Transactions on Ultrasonics, Ferroelectrics, and Frequency Control, Vol.38 (1991), pp.89-92.

(5) Kentaro, N., Kurosawa, M. and Ueha, S., Chracteristics of a hybrid transducer type ultra-sonic motor, IEEE Transactions on Ultrasonics, Ferroelectrics and Frequency Control, Vol.38 (1991), pp.188-193.

(6) Takano, T., Tomikawa, Y. and Kusakabe, C., Same phase drive-type ultrasonic motors using two degenerate bending vibration modes of a disk, IEEE Transactions on Ultrasonics, Ferroelectrics, and Frequency Control, Vol.39 (1992), pp.180-186.

(7) Nesbitt, W., Hagood, IV and Andrew, J., Modeling of a piezoelectric rotary ultrasonic motor, IEEE Transactions on Ultrasonics, Ferroelectrics, and Frequency Control, Vol.42 (1995), pp.210-223.

(8) Nicola, L., Antonio, I. and Massimo, P., A piezoelectric motor using flexural vibration of a thin piezoelectric mem-brane, IEEE Transactions on Ultrasonics, Ferroelectrics, and Frequency Control, Vol.45 (1998), pp.23-29.

(9) Moal, P.L. and Cusim, P. Optimization of traveling wave ultrasonic motors using a three-dimensional analysis of the contact mechanism at the stator- rotor interface, Journal of Mechanics, A/Solids, Vol.18 (1991), pp. 1061-1084.

(10) Wen, F.L., Yen, C.Y. and Ouyang, M.S., Thin-disk piezoceramic ultrasonic motor. Part I: design and performance evaluation, Ultrasonics, Vol.41 (2003), pp.437-450.

(11) Yen, C.Y., Wen, F.L. and Ouyang, M.S. Thin-disc piezoceramic ultrasonic motor. Part II: system construction and control, Ultrasonics, Vol.41 (2003), pp.451-463.

(12) Bai, D., Ishii, T., Nakamura, K., Ueha, S., Yonezawa, T. and Takahashi, T., An ultrasonic motor driven by the phase-velocity difference between two traveling waves, IEEE Transactions on Ultrasonics, Ferroelectrics, and Frequency Control, Vol.51 (2004), pp.680-685.

(13) Flynn, A.M., Performance of ultrasonic mini-motors using design of experiments, Smart Materials and Structures, Vol.7 (1998), pp.286-294.

(14) Ville, K. and Amit, L., Optimization of a bulk-driven surface micro machined ultrasonic micromotor, IEEE Ultrasonics Symposium, Vol.1 (2001), pp.541544.

(15) Ishii, T., Maeno, S., Nakamura, K. and Ueha, S., Efficiency improvement of the friction drive in the ultrasonic motor using lubricant, IEEE Ultrasonics Symposium, Vol.1 (2001), pp.521-524.

(16) Pons, J.L., Rodriguez, H., Fernandez, J.F., Villegas, M. and Seco, F., Parametrical optimisation of ultrasonic motors, Sensors and Actuators, A: Physical, Vol.107 (2003), pp.169-182.

(17) Zhao, C.S., Li, Z. and Huang, W.Q., Optimal design of the stator of a three-DOF ultrasonic motor, Sensors and Actuators, A: Physical, Vol.121 (2005), pp.494-499.

(18) Xia, C.G. and Wang, M.G., Stability analysis of the rotor of ultrasonic motor driving fluid directly, Ultrasonics, Vol.43 (2005), pp.596-601.

(19) Shiau, T.N., Kang, C.H. and Liu, D.S, Multi-objective optimal design of rotor-bearing systems under dynamic behavior constraints using a hybrid genetic algorithm, Chinese Society of Mechanical Engineers, Vol. 29 (2008), pp. 187-194.

(20) Hollan, J.H., Outline for a logical theory of adaptive system, JACM (1962), pp.297-314.

(21) Hollan, J.H., Adaption in natural and artificial systems, University of Michigan Press, (1975).

(22) Goldberg, D.E., Genetic algorithms in search. Optimization \& Machine Leaning, Addision Wesley, New York, (1989).

(23) Gen, M. and Cheng, R., Genetic algorithms and engineering optimization, John 
Wiley \& Sons, INC, New York, (1999).

(24) Vanderpaats, G.N. Numerical optimization techniques for engineering design, McGraw-Hill, New York, (1993).

(25) Charnes, A. and Cooper, W., Management models and industrial application of linear programming, John Wesley \& Sons, New York, (1961). 